\title{
6 Siedlungen des Siebenstromlandes und ihre Zugehörigkeit zu den Zentralen Orten
}

Außer sakischen Nekropolen wurden im südöstlichen Siebenstromland einige Siedlungsplätze festgestellt, die möglicherweise in der Früheisenzeit angelegt worden waren.

Mit Ausnahme einiger weniger Untersuchungen einzelner Fundstellen528 (Grigor'ev I995; Chang/ Grigoriev I999; Bajpakov/Chang 2000; Chang/Tourtellotte 2000; Chang u.a. 2002; Chang u.a. 2003; Samašev u.a. 2005a, 52-53; Samašev u.a. 2005f; Gorjačev 2006; Bajpakov 2007; Baipakov 2008; Bajpakov 2008) bleiben Fragen zum sakischen Siedlungswesen völlig offen. Dass die Saken als Nomadenvolk nicht ausschließlich in zelt- oder jurtenartigen Behausungen oder in Wohn- und Planwagen lebten, wie es bei Herodot bezüglich der nordpontischen Skythen geschrieben steht (Herodot [4-46], 296), darf heute nicht mehr bezweifelt werden (Parzinger 2004, 56, 78-79; Gass 20IIb, 2I7). Mehrere Beweise dazu finden sich in den westlich der Saken gelegenen Gebiete, nämlich im nordpontischen Raum und am mittleren Dneprlauf. Die skythische Wallburg Bel'sk beispielsweise (Grakow I978, 49, 96-98, I42, I44-I49; Šramko I987; Parzinger 2004, 79), die im 5.-4. Jh. v. Chr. entstand (Parzinger 2004, 79), wurde als die von Herodot erwähnte Stadt Gelonos (Herodot [4-I08], 320) interpretiert. Weitere Beispiele sind die Kamenskoe Gorodišče (Grakov I954; Avdusin I977, I35; Parzinger 2004, 79-8I), die Gorodišče Širokaja Balka (Rusjaeva/Mazarati I986; Parzinger 2004, 8I) oder die spätskythische Stadt Neapolis Scythicae auf der Krim (Grakow I978, 9, 27, 34-35, 66-67; Zajcev 2003).

Jüngst wurde in Kasachstan die These entwickelt, dass die bekannten mittelalterlichen Siedlungsplätze im südöstlichen Siebenstromland an solchen Stellen errichtet wurden, die bereits in der Früheisenzeit besiedelt waren (Bajpakov u.a. 2002, 8-9; Baipakov 2008, I37-I38). Trifft diese Aussage zu, so könnte dies zumindest bedeuten, dass bei der Siedlungsplatzauswahl sowohl im Mittelalter als auch in der Früheisenzeit vergleichbare Kriterien gegolten haben könnten. Infolgedessen wurden bereits bekannte „mittelalterliche“ Fundplätze erneut untersucht. Im Zuge dieser Untersuchungen wurden auch neue Fundstellen entdeckt. Insgesamt wurden 22 Siedlungsplätze festgestellt (siehe Verzeichnis der früheisenzeitlichen Fundstellen mit Quellennachweis; Karte I9). Fünf davon waren Bestandteil sogenannter archäologischer Komplexe529.

Dabei handelte es sich um befestigte und unbefestigte Siedlungsplätze. Im Zentrum der befestigten Siedlungen können sich ein530 oder drei53 mächtige trapezförmige Hügel532 erheben (Abb. 99). Die Befestigung wird von Schutzwällen und Schutzgräben, die künstlich aus den Erosionsschluchten nachbearbeitet wurden, gebildet. Andere Anlagen bestehen aus einem viereckigen Wall-Graben-System, dessen Ecken oder Seiten an den vier Haupthimmelsrichtungen orientiert wurden (Abb. Ioo). In Mittelasien wurden Siedlungsplätze dieser Art als Turtkul (kasach. торткол, russ. турткуль533 оder торткуль534) bezeichnet. Der Innenraum des Turtkul wurde immer sorgfältig planiert und weist nie

528 Siedlungsbereiche der archäologischen Komplexe Butakty I und Turgen-2, Siedlungen Cyganka 8 und Tuzusaj.

529 Als archäologischer Komplex werden eine zeitgleiche Siedlung und ein Gräberfeld verstanden, die sich in unmittelbarer räumlicher Nähe befinden.

530 Siedlung Rachat (siehe Katalog; Karte 3 Nr. I2).
53I Siedlung Krasnyj Vostok (siehe Katalog; Karte 3 Nr. 13).

532 Der größte und höchste Hügel sollte wohl eine „Zitadelle" darstellen.

533 Schreibweise nach A. N. Bernštam (Bernštam I938, 7, $42)$.

534 Schreibweise nach K. M. Bajpakov (Bajpakov I978). 
Abb. 99 | Befestigte Siedlung Rachat. Überblick von $\mathrm{N}$

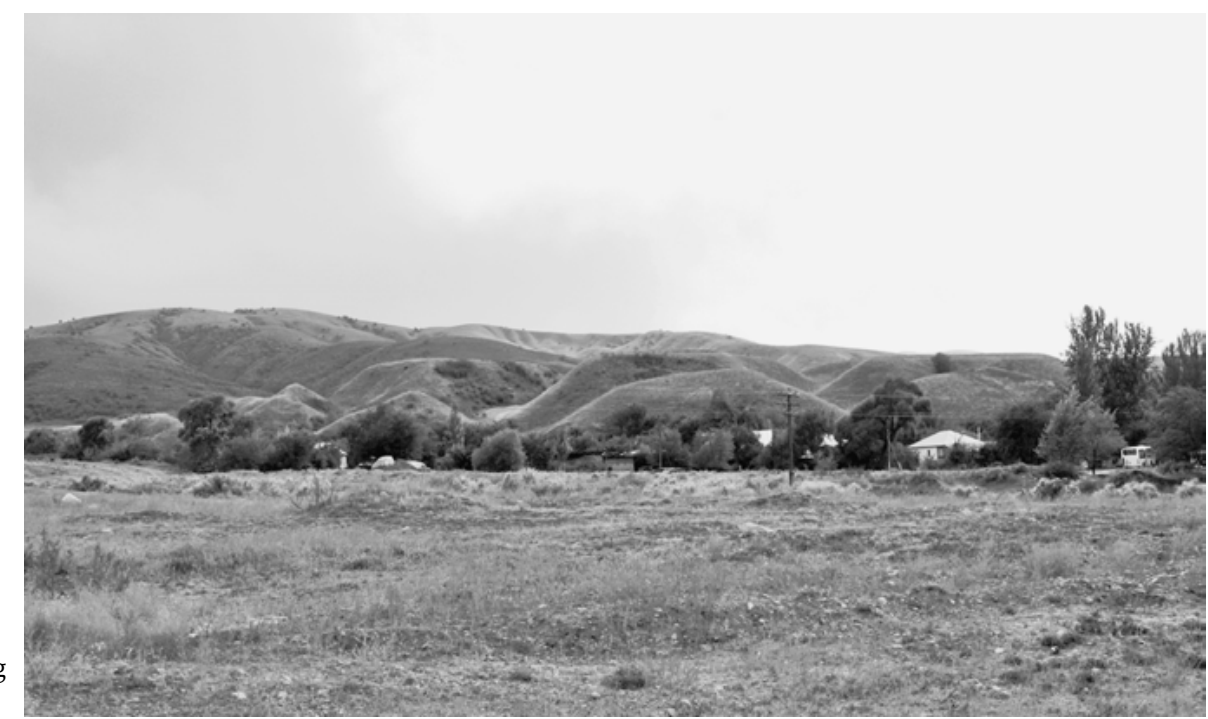

Abb. 100 | Komplex Kiikpaj. Skizze des Turtkuls

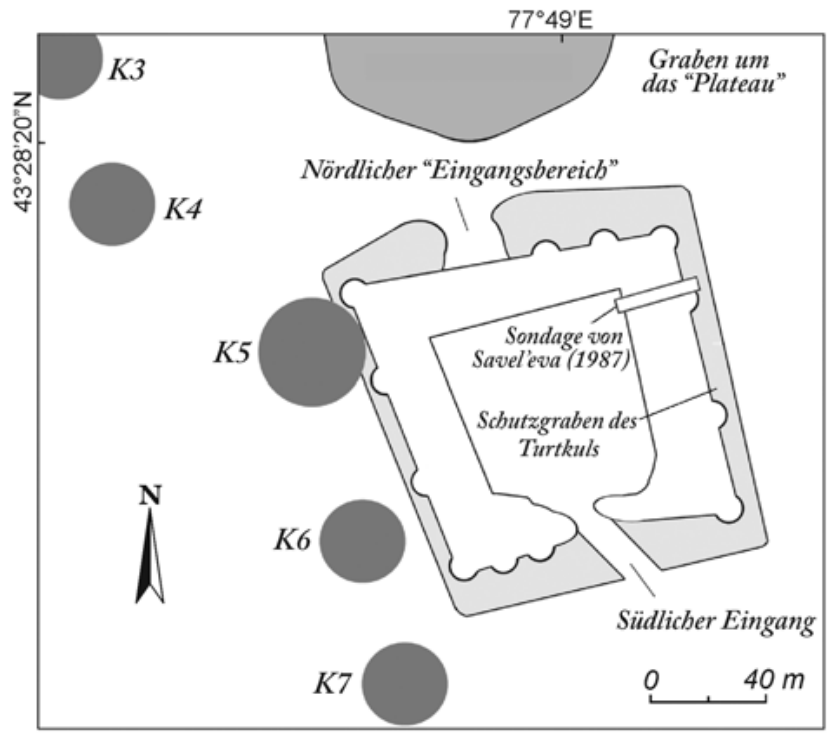

einen zitadellenartigen Bereich auf. Im südöstlichen Siebenstromland sind solche Anlagen aus dem Mittelalter bekannt und repräsentieren häufig die letzte Nutzungsphase des Siedlungsplatzes (Bernštam I938, 7, 42; Bajpakov I978; Savel'eva I994, 37, 44, 48, 56-60; Bajpakov u.a. 2002, 29, 40-4I, 6I-62). Auf den Schutzwällen der zuerst beschriebenen Befestigungsanlagen sowie auf den Wällen und an den Ecken der Turtkuls befinden sich kegelförmige Erhebungen, die möglicherweise Überreste von Wachtürmen darstellen. Sowohl auf der Kuppe der trapezförmigen Hügel der ersten Befestigungen als auch im Innenraum der Turtkuls kommen rechteckige oder ovale Gruben sowie Steinkonzentrationen oder abgerundete Erhebungen vor, die bislang nicht genauer angesprochen werden können. Alle befestigten Siedlungen befinden sich in der Nähe von fließenden Gewässern. Man muss jedoch noch zusätzlich vermerken, dass die Frage, ob befestigte Siedlungen jeglicher Art bereits in der Früheisenzeit umbaut waren oder nicht, anhand heutiger Kenntnisse des Siedlungswesens im Forschungsgebiet noch ungeklärt bleiben muss. 


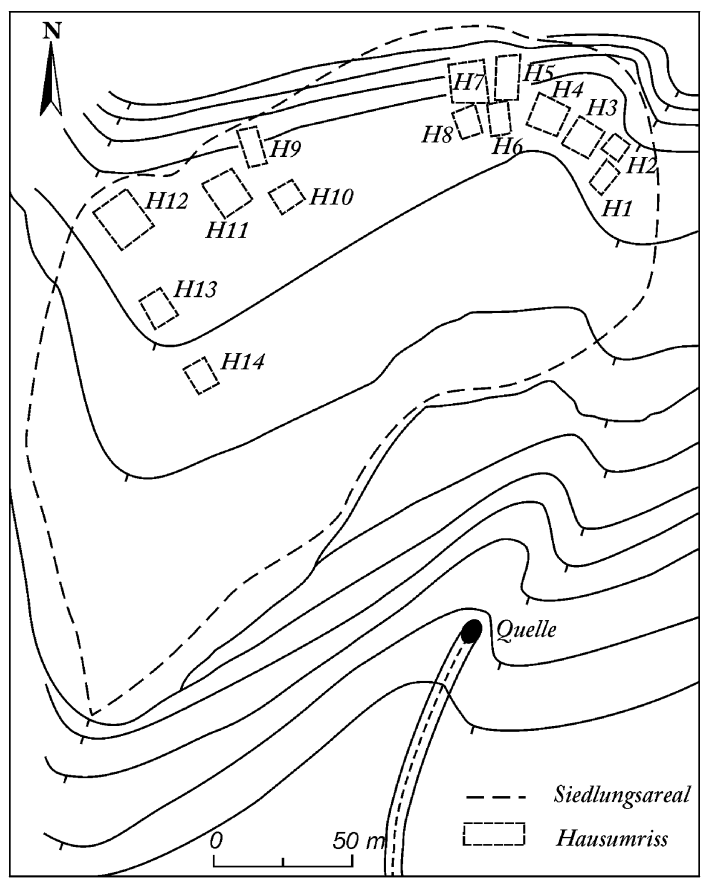

Abb. 101 | Unbefestigte Siedlung Kotlovandy. Lageskizze

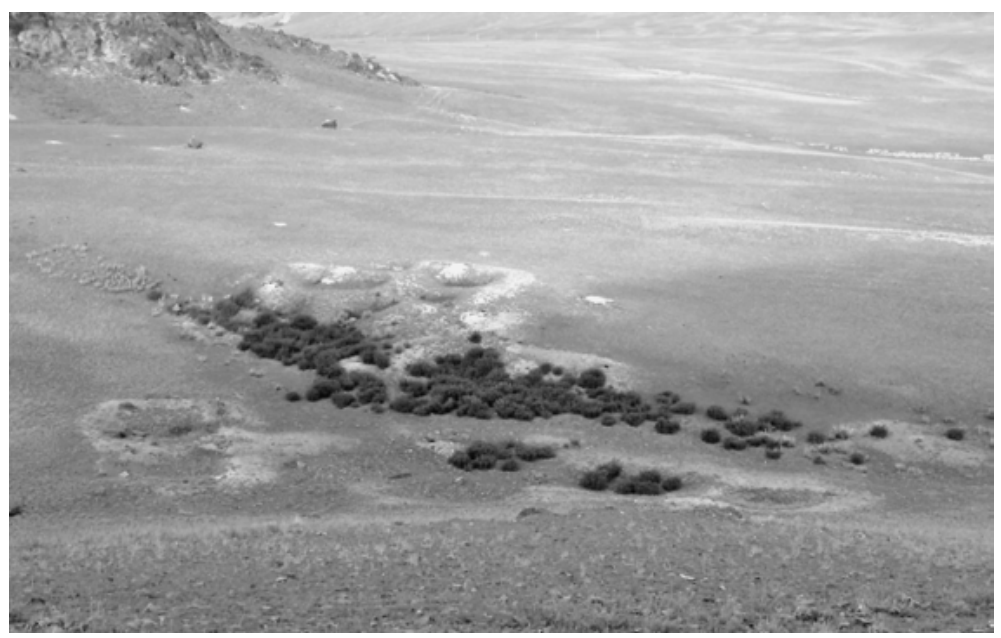

Abb. 102 | Unbefestigte Siedlung Sarytau.

Überblick von W

Unbefestigte Siedlungsplätze, die nicht an Flussufern, sondern in kleinen Bergschluchten liegen und dadurch von drei Seiten durch Berghänge geschützt sind, weisen fast nie eine sichtbare, äußere Begrenzung auf535. Die innere Struktur solcher Niederlassungen unterscheidet sich ebenfalls von denen der flussnahen Siedlungen. Sie bestehen aus zwei oder drei Hausreihen, von denen heute noch rechteckige, steinerne Überresten der Fundamente von „Doppelwandhäusern“ oder anderen Subkonstruktionen eines Rechteckhauses sichtbar sind. Ovale Gruben werden als sog. Poluzemljanki (russ.) interpretiert (Abb. IOI-IO2).

535 Als eine Ausnahme kann hier die Siedlungsfläche des archäologischen Komplexes Sarytau genannt werden (siehe Abb. 224, 225,2-226). Hier wurde ein von den Berghängen und der Felsenkuppe nicht geschützter Ab- schnitt durch eine doppelte Steinreihe markiert, die eventuell als Fundament einer äußeren „Umfriedung“ dienen könnte. 
Die Kartierung der Siedlungsplätze zeigte, dass sich im südöstlichen Siebenstromland drei Verbreitungsschwerpunkte abzeichnen (Karte 20) und bei der Siedlungsplatzauswahl in jeder Verbreitungszone immer gleiche Landschaftsmarker von Bedeutung waren.

Die erste Verbreitungszone befand sich an der Nordseite des Transili-Alatau, im Übergangsbereich vom Lösshügelland in den Schwemmfächer (7I4-I203 m ü NN). Dort herrschen kontinentales Klima sowie Tschernosem- und Kastanozem-Böden vor (Karte 5; vgl. auch Kap. 2). Siedlungen und sakische Gräberfelder nehmen prinzipiell räumlich Bezug aufeinander. Kennzeichnend ist jedoch, dass sich die Nekropolen weiter nördlich in der Ebene befanden und die Siedlungsplätze südlich, nämlich exakt am Fuße der Berghänge, wo die Bergflüsse in die Flachebene übergehen, an den Ufern der Flüsse. Durch diese Lage war ein hervorragender natürlicher Schutz gewährleistet und die Verteidigung der Anlagen vereinfacht. Da zumeist Plätze ausgesucht wurden, die von drei Seiten durch Flüsse und Berge umgeben waren, musste nur die vierte, leicht begehbare Seite verteidigt werden (Gass 20IIb, 2I7). Zudem verlief die westöstlich orientierte Verbreitungsgrenze der Siedlungsplätze nicht so weit nach Osten wie die der Nekropolen. Die westliche Grenze markierte die Siedlung des archäologischen Komplexes Butakty ${ }_{53} 6 \mathrm{im}$ Bereich der modernen Stadt Almaty, die östliche der Siedlungsplatz Junki-I537 in der Nähe des Dorfes Teskensu, 8 km südöstlich vom Gräberfeld Bes Tobe538. Die gesamte Strecke maß $80 \mathrm{~km}$ Luftlinie und umfasste I6 Siedlungsplätze, die zumeist durch Lesefunde an der Oberfläche als mögliche, früheisenzeitliche Wohnplätze angesprochen werden konnten. Die Siedlungen waren zwischen I,I km539 und I9,० km540 voneinander entfernt. Der durchschnittliche Abstand zwischen den Siedlungen betrug 6,7 km. Vom nächstgelegenen Gräberfeld54 lagen die Ansiedlungen zwischen I, 8 km542 und I4,I km543 entfernt. Der durchschnittliche Abstand zwischen einem Siedlungsplatz und einem Gräberfeld maß $7 \mathrm{~km}$. Wie die Tabelle 28 und die Abbildung I03 zeigen, war für die Siedlungsplatzwahl der Übergangsbereich vom Lösshügelland ins Flachland, am Ufer eines fließenden Gewässers eine unbedingte Voraussetzung. Dabei spielte die Flussseite beim Errichten eines Siedlungsplatzes offensichtlich keine so große Rolle. Es wurden sieben Siedlungen (44\% aller untersuchten Siedlungsplätze in der ersten Verbreitungszone) am linken westlichen Ufer eines Flusses bzw. eines Baches gebaut und sechs (37\%) am rechten östlichen Ufer. Weitere drei Siedlungsplätze (I9\%) befanden sich zwischen zwei Wasserläufen, zwischen einem Fluss und einem Bach oder zwischen zwei Bächen. Insgesamt wurde also bei der Wahl des Platzes Wert gelegt auf das Vorhandensein eines Wasserlaufes und auf die durch die Landschaftsbeschaffenheit vorgegebenen, natürlichen Schutzbedingungen.

Die Siedlungen der zweiten Verbreitungszone liegen in kleinen Seitenschluchten des alpinen Bereiches der Schlucht Turgen oberhalb der Waldgrenze (2336-2475 m ü NN). Dies entspricht dem „4. Lebensraum - Wald- bzw. Wiesenzone“ (Abolin I930, I7I-I72; siehe Kap. 2. Geographische Lage und klimatische Verhältnisse des Untersuchungsgebietes), in dem ein nivales Klima (Karte 5) und „Tschernosemböden der Gebirgswiese“ vorherrschen (Abolin I930, I7I). Die Siedlungsplätze der we-

536 Karte 3 und Karte 20 jeweils Nr. 5.

537 Karte 3 und Karte 20 jeweils Nr. 25.

538 Karte 3 Nr. 22.

539 Abstand zwischen den Siedlungsplätzen Rachat (Karte 20 Nr. I2) und Krasnyj Vostok (Karte 20 Nr. I3).

540 Abstand zwischen den Siedlungen des archäologischen Komplexes Butakty I (Karte 20 Nr. 5) und Kyzyl-Gajrat (Karte 20 Nr. 8).

54I Hier wurden nur Entfernungsparameter berücksichtigt, da es unklar ist, ob die entsprechenden Nekropolen und die Siedlungsplätze tatsächlich in Bezug zueinander standen. Dies könnte nur durch künftige Ausgrabungen beider Fundorte geklärt werden.

542 Abstand zwischen der Siedlung Krasnyj Vostok (Karte 3 Nr. 13) und dem Gräberfeld Issyk (Karte 3 Nr. I4).

543 Abstand zwischen dem Siedlungsplatz des archäologischen Komplexes Butakty I (Karte 3 Nr. 5) und der Nekropole Ulžan (Karte 3 Nr. 3 ). 
Tabelle 28 | Verhältnis der untersuchten Niederlassungen zu den fließenden Gewässern der ersten Verbreitungszone (Fundorte geographisch von Westen nach Osten aufgeführt)

\begin{tabular}{|l|l|l|l|l|}
\hline Siedlungsplatz & Befestigung & Gewässer & Uferseite & Ufer \\
\hline Butakty I & keine & Fluss Žarbulak (Kazačka) & rechte & O \\
\hline Tuzusaj & keine & ausgetrockneter Fluss Tuzusaj & linke & W \\
\hline Cyganka 8 & keine & Fluss Cyganka & rechte & O \\
\hline Kyzyl-Gajrat & Turtkul & zwischen dem ausgetrochneten Flussbett & linke / rechte & W / O \\
\hline Talgar & Turtkul einem Bach & rechte & O \\
\hline Kajnazar & keine & Fluss Talgar & linke & W \\
\hline Rachat & Wälle und Schutzgräben & Fluss Kajnazar & linke & W \\
\hline Krasnyj Vostok & Wälle und Schutzgräben & Bergfluss & rechte & O \\
\hline $\begin{array}{l}\text { Siedlungsstelle bei der } \\
\text { Stadt Issyk }\end{array}$ & Wall & Fluss Esik & linke & W \\
\hline $\begin{array}{l}\text { Issyk „Zentraler Fried- } \\
\text { hof“ }\end{array}$ & Wall & & linke / rechte & W / O \\
\hline $\begin{array}{l}\text { Issyk „Tagebaustätte } \\
\text { beim Geburtshaus“ }\end{array}$ & keine & zwischen zwei Bächen & rechte & O \\
\hline Karakemer & Turtkul & Bach & linke & W \\
\hline Bachtijar & Turtkul mit Schutzgraben & Fluss Bachtijar & rechte & O \\
\hline Aščibulak & Turtkul & Fluss Kury-Aščbulak & linke & W \\
\hline Kiikpaj & Turtkul mit Schutzgraben & Fluss Kiikbaj & linke & W \\
\hline Junki-I & keine (?)544 & zwischen dem Fluss Junki und einem Bach & rechte / linke & O / W \\
\hline
\end{tabular}

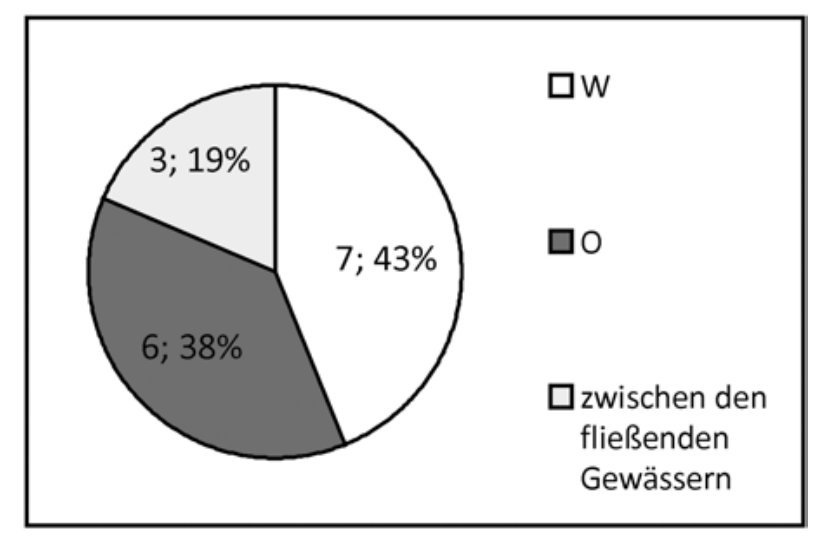

Abb. 103 | Verhältnis der Platzierung der untersuchten Niederlassungen der ersten Verbreitungszone bezüglich des Flussverlaufs und der Himmelsrichtungen

sentlich kleineren, zweiten Verbreitungszone (Karte 20) lagen an den Stellen, die von drei Seiten durch Berghänge geschützt waren und die vierte Seite an einen Bach bzw. eine Quelle grenzte. Drei Siedlungsplätze dieser Verbreitungszone waren voneinander zwischen 2,8 km545 und I,7 km546 entfernt. In der zweiten Verbreitungszone wies lediglich der Archäologische Komplex Turgen-2 einen Bezug zwi-

544 Siehe Katalog, Siedlung Junki-I.

545 Abstand zwischen der Siedlung Kotlovandy (Karte 20 Nr. 30) und dem Siedlungsplatz des archäologischen Komplexes Turgen-2 (Karte 20 Nr. 33).
546 Abstand zwischen den Siedlungen des archäologischen Komplexes Turgen-2 (Karte 20 Nr. 33) und Kyzylbulak-2 (Karte 20 Nr. 34). 
Tabelle 29 | Verhältnis der untersuchten Niederlassungen zu den fließenden Gewässern der zweiten Verbreitungszone (Fundorte geographisch von Nordwesten nach Südosten aufgeführt)

\begin{tabular}{|l|l|l|l|l|}
\hline Siedlungsplatz & Befestigung & Gewässer & Uferseite & Ufer \\
\hline Kotlovandy & keine & Quelle & keine & $\mathrm{N}$ \\
\hline Turgen-2 & keine & Bach Kyzylbulak & rechte & NW \\
\hline Kyzylbulak-2 & keine & Bach Kyzylbulak & rechte & NO \\
\hline
\end{tabular}

schen Siedlung und Nekropole auf, so dass die Informationen bezüglich der Entfernung zwischen Gräberfeld und Siedlung in dieser Zone für eine Analyse zu gering ausfallen. Zudem soll es hervorgehoben werden, dass in diesem Bereich keine Gräberfelder mit sakischen Großkurganen festgestellt werden konnten.

Wie Tabelle 29 zeigt, liegen alle Siedlungen der zweiten Verbreitungszone am nördlichen rechten Ufer, falls eines vorhanden war. In der Nähe der Siedlung Kotlovandy gab es keinen Fluss oder Bach, dafür befand sich die Niederlassung nördlich einer Wasserquelle. Folglich wurde eine ganze Reihe von Siedlungsflächen in der zweiten Zone nördlich von Gewässern lokalisiert.

Die dritte Konzentration früheisenzeitlicher Siedlungen wurde an der Ostgrenze des Hochplateaus Kegen (I872-I956 m ü NN) festgestellt. Hier herrscht ein nivales Klima vor (Karte 5) und der Boden ist durch Tschernoseme und Kastanozeme charakterisiert (siehe Kap. 2. Geographische Lage und klimatische Verhältnisse des Untersuchungsgebietes). Die Siedlungsplatzwahl erfolgte nach ähnlichen Kriterien wie in der zweiten Verbreitungszone. Die Wohnplätze befanden sich auch hier in kleinen Bergschluchten, so dass die Siedlungen von drei Seiten durch Berghänge geschützt waren. Allerdings besteht ein entscheidender Unterschied darin, dass in der Nähe der Siedlungen der dritten Zone keine Gewässer festgestellt wurden. Die nächstgelegenen Flüsse fanden sich erst in einem Abstand von I,o bis $2,3 \mathrm{~km}$ von den Niederlassungen entfernt. Selbst eine Entfernung von I km stellt für eine ununterbrochene Wasserversorgung einer ganzen Siedlungsgemeinschaft eine nicht geringe Schwierigkeit dar. Die Frage nach der Wasserversorgung der Siedlung muss zunächst ungeklärt bleiben. Die Siedlungsplätze547 der dritten Verbreitungszone waren zwischen I,3 km548 und 4,I km549 voneinander entfernt. Die zugehörigen Gräberfelder lagen 2,3 km550 bis 3,I55I-3,8552 km entfernt; nur die Nekropole des Archäologischen Komplex Sarytau war mit I4-450 m sehr nahe. Die Siedlungen Aktasty und Sarytau liegen auf einer Seite des Berges und das Gräberfeld Aktasty-3 und die Kurgankette Sarytau „A“ auf der anderen Seite desselben Berges. Zwischen der Siedlung Sarytau und dem Kurgan 2 des Gräberfeldes auf dem Plateau Kegen fließt der Fluss Kegen. Daher konnte das Merkmal Entfernung zwischen einem Siedlungsplatz und einer Nekropole nur bedingt analysiert werden, da nicht bewiesen ist, ob die Fund-

547 Von Westen nach Osten aufgeführt: Siedlung Sarytau (Karte 20 Nr. 7I), Siedlungsfläche des Archäologischen Komplexes Sarytau (Karte 20 Nr. 72) und Siedlung Aktasty (Karte $20 \mathrm{Nr} .73)$.

548 Abstand zwischen der Siedlung Sarytau (Karte 20 Nr. 7I) und dem Siedlungsplatz des Archäologischen Kompexes Sarytau (Karte 20 Nr. 72).

549 Abstand zwischen den Siedlungen des archäologischen Komplexes Sarytau (Karte 20 Nr. 72) und Aktasty (Karte 20 Nr. 73).
550 Abstand zwischen der Siedlung Aktasty (Karte 3 Nr. 73) und dem Gräberfeld Aktasty-3 (Karte 3 Nr. 74).

55I Abstand zwischen der Siedlung Sarytau (Karte 3 Nr. 7I) und der Kurgankette Sarytau "A“ (Karte 3 Nr. 70).

552 Abstand zwischen der Siedlung Sarytau (Karte 3 Nr. 73) und dem Kurgan 2 der Nekropole auf dem Plateau Kegen (Karte 3 Nr. 68). 
orte tatsächlich in Bezug zueinander stehen. Eine Analyse der Siedlungsplatzwahl für die dritte Verbreitungszone bezüglich der Gewässer ist ebenso nicht möglich.

Zur äußeren Form sowie zur Funktion der Siedlungen konnte festgestellt werden, dass sowohl befestigte, trapezförmige Siedlungshügel, die von Schutzwällen und Schutzgräben umgeben waren (Rachat (Abb. 99) und Krasnyj Vostok), als auch befestigte Turtkuls (Abb. Ioo) nur in der ersten Verbreitungszone vorkommen. Innerhalb dieser Siedlungen wurden Steinkonzentrationen sowie rechteckige bzw. ovale Gruben oder abgerundete Erhebungen festgestellt. Angaben zur ehemaligen Form und Interpretation der Befunde sind zum gegenwärtigen Forschungsstand nicht möglich.

Die Siedlungen Rachat und Krasnyj Vostok wurden von den Forschern in ihrer ersten Phase mit den Trägern der sakischen Kultur verbunden und beiden Ansiedlungen wurde die Rolle eines Zufluchtsortes und eines politischen Zentrums zugesprochen (Bajpakov/Nurmuchanbetov 2000, I5-I6; Baipakov 2008, 72; siehe Katalog, Siedlungen Rachat und Krasnyj Vostok, Forschungsgeschichte). Die Turtkuls wurden im Mittelalter wohl als Karawansereien benutzt (Bernštam I938, 42). Wie solche Siedlungsplätze in der Früheisenzeit553 aussahen und welche Funktion sie hatten, bleibt zunächst ungeklärt, da solche Fundorte nur selten und meist unvollständig untersucht wurden. Selbst grundlegende Informationen fehlen.

Fast alle unbefestigten Siedlungsplätze der ersten Verbreitungszone wiesen an der Oberfläche gar keine Befunde auf. Eine Ausnahme stellte die Siedlungsstelle Issyk „Tagebaustätte beim Geburtshaus“ dar (siehe Katalog), wo bereits an der Oberfläche vier abgerundete Gruben mit einem Durchmesser von 4-8 m festgestellt wurden. Es könnte sich hier um Überreste von Gruben- bzw. Halbgrubenhäusern (russ. Poluzemljanki) handeln.

Im Gegensatz zu den unbefestigten Siedlungen der ersten Verbreitungszone wiesen die inneren Bereiche der Siedlungsplätze der zweiten und der dritten Verbreitungszone immer einige Befunde an der modernen Oberfläche auf. Dabei handelte es sich um fünf bis zwölf rechteckige Gruben554 bzw. in den Berghang eingegrabene, planierte, rechteckige Flächen, deren äußere Grenze von einzelnen Steinen markiert wurde555. Solche Steine können als Fundamentreste von Gebäuden angesprochen werden. Einige dieser Konstruktionen wurden quer oder längs durch eine zusätzliche Steinreihe bzw. kleine Erdwälle in zwei556 bis sechs Parzellen bzw. Räume557 unterteilt. Ferner traten zu einem Rechteck gelegte Steinreihen auf558, die möglicherweise eine Subkonstruktion eines Rechteckhauses darstellten. Ein Rechteck bildende Doppelsteinreihen können evtl. als Fundamentreste der Doppelwandhäuser interpretiert werden.

Die erwähnten Konstruktionen bilden „Hausreihen“. Sowohl die einzelnen Bauten als auch die „Hausreihen“ wurden unterschiedlich ausgerichtet. Die Orientierung der Bauten war vollständig von

553 Dafür, dass die Fundorte bereits in der Früheisenzeit besiedelt wurden, spricht die von der Oberfläche aufgelesene, früheisenzeitliche Keramik (siehe Katalog: Siedlung Kyzyl-Gajrat, Charakteristik der Lesefunde, Abb. 262; Siedlung Talgar, Siedlungscharakteristik und Forschungsgeschichte, Abb. 269; Siedlung Issyk „Zentraler Friedhof", Charakteristik der Lesefunde, Abb. 244; Siedlung Karakemer, Charakteristik der Lesefunde, Abb. 253; Siedlung Bachtijar, Charakteristik der Lesefunde, Abb. 235; Komplex Aščibulak, Charakteristik der Lesefunde, Abb. 2I5 und Komplex Kiikpaj, Charakteristik der Lesefunde, Abb. 220).
554 z.B.: siehe Katalog Siedlung Kotlovandy, Abb. Ior und Abb. 255, Charakteristik der „Häuser“; Siedlung Sarytau, Abb. IO2 und Abb. 267; Komplex Sarytau, Abb. 225,2, Charakteristik der Häuser oder Siedlung Aktasty, Abb. 23I,2.

555 z.B.: siehe Katalog Siedlung Kotlovandy, Abb. Ioı, Charakteristik der "Häuser“ oder Siedlung Kyzylbulak-2, Abb. 260, Charakteristik der „Häuser“.

556 z.B.: siehe Katalog Siedlung Kyzylbulak-2, Abb. 259; Siedlung Sarytau, Abb. IO2 und Abb. 266-267 oder Siedlung Aktasty, Abb. 230.

557 z. B.: siehe Katalog Siedlung Kyzylbulak-2, Abb. 259.

558 z.B.: siehe Katalog Komplex Sarytau, Charakteristik der Häuser. 

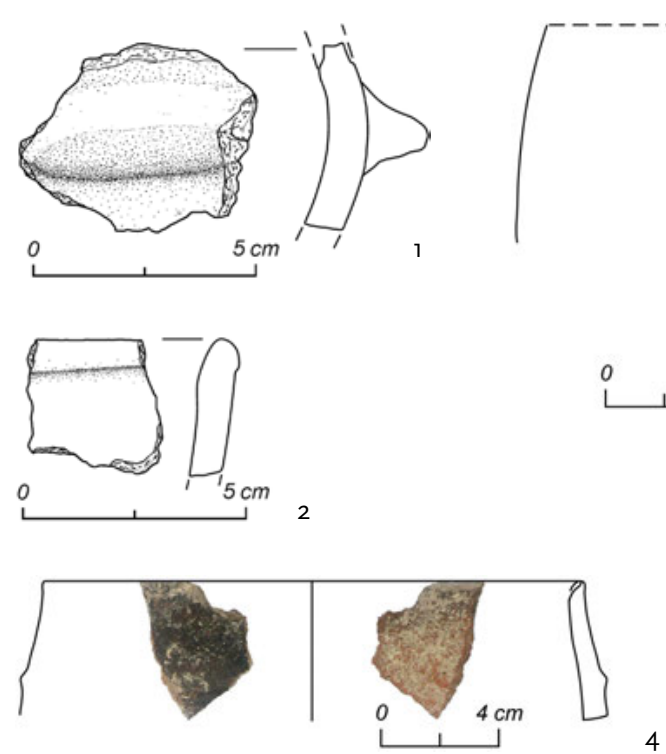

4
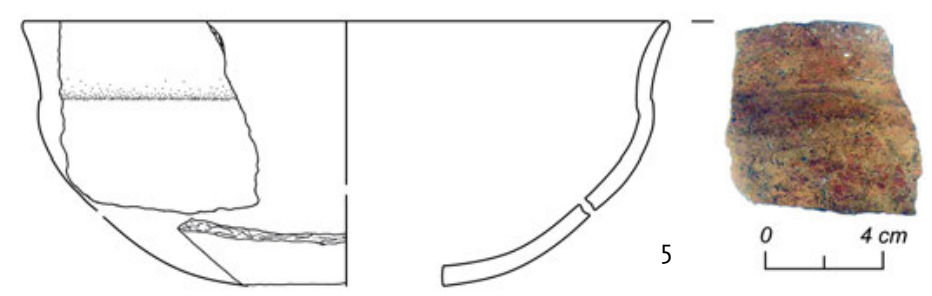

Abb. 104 | Lesefunde von der Oberfläche. Früheisenzeitliche Keramik (I - WS mit Griff; 2, 4-5 - RS; 3- Henkel; 6-7 - WS / I-2 - Sdl. Aktasty; 3 - Sdl. Karakemer; 4-7 - Sdl. Tuzusaj) [Foto: A. Gass; Zeichnung: M.-R. Boroffka]

den naturräumlichen Begebenheiten abhängig. Zudem muss immer bedacht werden, dass die festgestellten Hausüberreste zu größeren, komplexeren Konstruktionen gehört haben könnten. Genaue Informationen dazu können jedoch nur Ausgrabungen erbringen.

Bezüglich des Ausmaßes der Siedlungsflächen konnten keine aussagekräftigen Angaben gemacht werden, da in der Regel die äußere Siedlungsgrenze nur anhand der sichtbaren Konstruktionen bzw. der Keramikstreuung an der Oberfläche bestimmt wurde. Dies kann zunächst nur eine ungefähre Angabe bleiben. Die tatsächliche Flächengröße könnte durch weitere Prospektionen oder Ausgrabungen festgestellt werden.

An der Oberfläche mehrerer Siedlungsflächen wurden Lesefunde (Abb. I04) aufgenommen, die eine relativchronologische Datierung dieser Fundorte ermöglichen. Die Lesefunde kamen nicht nur auf den unbefestigten Siedlungsplätzen aller drei Verbreitungszonen, sondern auch in den Turtkuls der ersten Verbreitungszone zum Vorschein. Es handelte sich dabei vor allem um unterschiedliche Keramikfragmente, die eine relativchronologische Tiefe von der Früheisenzeit bis zum Mittelalter aufzeigten. Diese Tatsache lässt vermuten, dass die Siedlungsplätze wiederholt und in unterschiedlichen Zeitperioden aufgesucht wurden. Die Früheisenzeit wurde hier durch rötliche (dunkel- und hellrot), beige und orangefarbene, mittelgrob- bis feingemagerte Keramik repräsentiert. Die Keramik wurde in einigen Fällen außen geglättet. Es handelt sich fast ausschließlich um unverzierte Drehscheibenkeramik. 

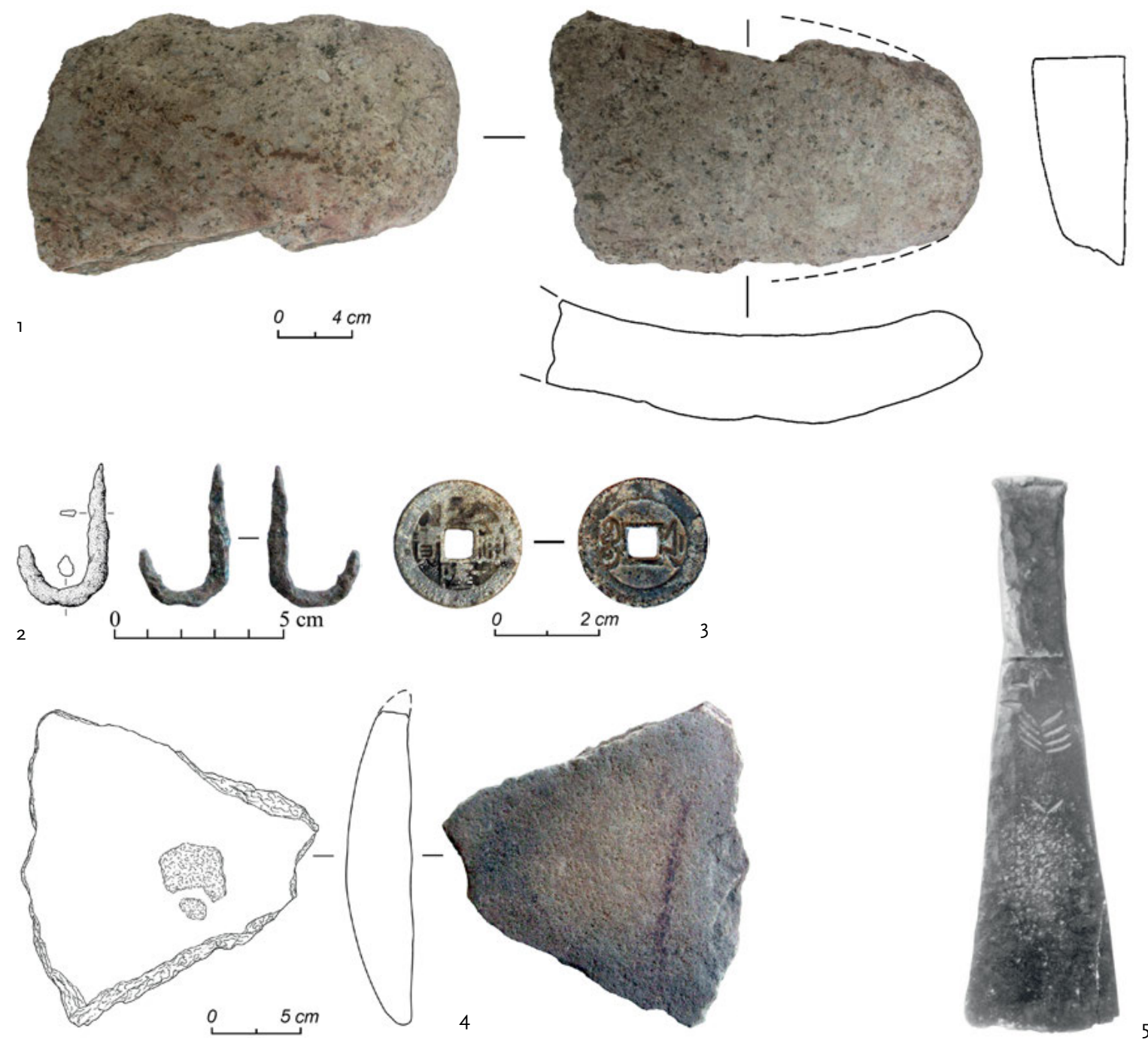

Abb. 105 | Lesefunde von der Oberfläche der untersuchten Niederlassungen (I - Reibstein, Sdl. Kajnazar; 2 - eiserner Angelhaken, archäologischer Komplex Sarytau; 3 - chinesische Bronzemünze, Sdl. Aktasty; 4 - Mühlstein, Sdl. Aktasty; 5 - bronzener Meißel, Sdl. Talgar) [I-4 Foto: A. Gass; I-2, 4 Zeichnung: M.-R. Boroffka; 5 - Kopylov I956, Taf. I4,7]

Die Scherben stammen von zerbrochenen Krügen, Schalen, Näpfen und Töpfen. An einigen Fragmenten waren Henkel oder Griffe erhalten.

Zudem wurden auch Reib- (Abb. I05,I) und Mühlsteine (Abb. I05,4), Angelhaken (Abb. I05,2) und eine chinesische Bronzemünze (Abb. I05,3) aufgelesen. Wenngleich Gegenstände wie Reibsteine oder ein eiserner Angelhaken sowohl in der Früheisenzeit als auch in späteren Zeitperioden hergestellt und benutzt worden sein können, gehören Mühlsteine sicherlich in die ethnographische Zeit. Ein eindeutiges chronologisches Indiz lieferte die chinesische Bronzemünze, die relativ gut erhalten war. Anhand der erhaltenen Schriftzeichen konnte sie als eine Bao Zhe-Münze bzw. Käsch-Münze, die unter der Regierung des chinesischen Kaisers Qianlong zwischen 1756 und 1796 extra für die Xinjang-Provinz geprägt wurde (Jen 2000, I89-190), bestimmt werden. Diese Funde beweisen zusätzlich eine wiederholte Nutzung der Siedlungen im südöstlichen Siebenstromland. 
Von besonderem Interesse sind ein bronzener Meißel (Abb. I05,5), ein sakischer Bronzekessel, eine Lanzenspitze aus Bronze sowie sakische Keramik, die auf der Siedlungsfläche und in den unteren Siedlungsschichten der Siedlung Talgar während der ersten Untersuchungen aufgehoben wurden (Gorodeckij I924, II8-II9 Nr. 20; Kopylov I956, 32, Taf. I4,7; Kopylov I962, 26-27). Das heutige Aussehen eines Turtkuls erhielt die Siedlung während der mittelalterlichen Nutzungsphase (Kopylov I956; Savel'eva I994, 2I-33; Bajpakov u.a. 2002, 8-28, 60-I49). Allerdings ist denkbar, dass die mittelalterliche Stadt Talchir (Kopylov I978, 6I-65; Savel'eva I994, I37; Bajpakov u.a. 2002, II, 20) an der Stelle einer früheisenzeitlichen Siedlung der sakischen Periode entstand (Kopylov 1956, 32; Kopylov I962, 47-48; Kopylov i965, 56-57; Kopylov I982, 22-23).

Ferner sollen zwei Suchschnitte $(2 \times 2 \times 0,7 \mathrm{~m}$ und $4 \times 4 \times 2,95 \mathrm{~m})$ auf der "Zitadelle“ der Siedlung Rachat in der Nähe der modernen Stadt Issyk erwähnt werden559. Außer Asche und Knochen wurden hier sowohl mittelalterliche als auch früheisenzeitliche Keramikfragmente freigelegt (Bajpakov u.a. 2005c, 4-5 Foto 4-5, 9 Abb. I-2, 6). „Die Lesefunde von der Oberfläche des Siedlungsplatzes und von den Ufern des Rachat-Flusses in der Nähe der Siedlung gehörten überwiegend der sakischen Periode an“ (Baipakov 2008, I46-I47). Es ist also anzunehmen, dass die Siedlungsstelle Rachat schon seit der Früheisenzeit existierte und wahrscheinlich mit einigen Unterbrechungen bis zum Mittelalter weiter genutzt wurde (Gass 20IIb, 2I8-219).

Bisher wurden hier nur Lesefunde und einige kleinflächige Untersuchungen von Siedlungsflächen des südöstlichen Siebenstromlands erwähnt, die möglicherweise bereits in der Früheisenzeit existierten. Tabelle 30 listet teilweise erforschte Siedlungsplätze auf, die gezielt auf die Frage nach dem Siedlungswesen der frühen Eisenzeit im Siebenstromland hin untersucht wurden.

Die in der Tabelle 30 erwähnten Siedlungsplätze wurden nur zum Teil ausgegraben. Daher bleiben Fragen nach dem Ausmaß der früheisenzeitlichen Siedlungen im südöstlichen Siebenstromland sowie nach der Innenbebauung zunächst offen. Die untersuchten Flächen der ersten Verbreitungszone wiesen abgerundete oder ovale bis rechteckige Gruben- und Halbgrubenhäuser sowie jurtenartige Häuser auf. In der zweiten Verbreitungszone handelte es sich nur um ebenerdige Häuser. Zudem wurden Speicher-, Wirtschafts- und Müllgruben freigelegt. Alle untersuchten Hauskonstruktionen hatten ein geringes Ausmaß. Die rundlichen Bauten hatten einen Durchmesser von 3,6 $\mathrm{m}^{56 \circ}$ bis zu $7 \mathrm{~m}^{56 \mathrm{r}}$ und die rechteckigen Konstruktionen wiesen eine Größe von 6,o × 3,7 m562 bis zu $9 \times 8 \mathrm{~m}^{563}$ auf. Die Ausrichtung der ovalen und rechteckigen Konstruktionen war von den naturräumlichen Gegebenheiten abhängig. Am Rande der durch Steine angezeigten Hausfundamente und manchmal in der Mitte des Hausbereiches wurden Pfostenlöcher festgestellt, die in einigen Fällen noch zusätzlich durch Steinsetzungen verstärk worden waren.

Bei den jurtenartigen Häusern der früheisenzeitlichen Siedlung des archäologischen Komplexes Butakty I handelte es sich um abgerundete, o,2 $\mathrm{m}$ tiefe Gruben, die am Rand Pfostenlöcher aufwiesen. Beide Wohnbauten wurden als rundliche Hütten rekonstruiert, die möglicherweise aus mit Lehm ver-

559 Siehe Katalog Siedlung Rachat.

560 z. B.: Grubenhaus 4 der Siedlung Cyganka 8; siehe Katalog Siedlung Cyganka 8, Charakteristik der Bauten.

56I Z. B.: jurtenartiges Haus 2 der früheisenzeitlichen Siedlung des archäologischen Komplexes Butakty I; siehe Baipakov 2008, 82 oder ebenerdiges Haus 2 der früheisenzeitlichen Niederlassung des archäologischen Komplexes Turgen 2; siehe Gorjačev 2004, 34 .
562 Z. B.: Grubenhaus 3 der Siedlung Cyganka 8; siehe Katalog Siedlung Cyganka 8, Charakteristik der Bauten.

563 Z. B.: ebenerdiges Haus I der früheisenzeitlichen Niederlassung auf dem archäologischen Komplex Turgen 2; siehe Gorjačev 200Ib, 22. 
Tabelle 30 | Teilweise untersuchte früheisenzeitliche Siedlungsplätze (Fundorte geographisch von Westen nach Osten aufgeführt)

\begin{tabular}{|c|c|c|c|}
\hline Siedlungsplatz & Untersuchter Bereich & Chronologische Einordnung ${ }^{564}$ & Quellenhinweis \\
\hline Butakty ${ }^{565}$ & zwei eingetiefte jurtenartige Häuser & 2. Hälfte des I. Jt. v. Chr. & $\begin{array}{l}\text { Baipakov } 2008,8 \mathrm{I}-88 \text {, } \\
\text { Abb. 30-32 }\end{array}$ \\
\hline Tuzusaj566 & $\begin{array}{l}\text { sieben Wirtschaftsgruben, zehn recht- } \\
\text { eckige Gruben- und Halbgrubenhäuser so- } \\
\text { wie die Überreste von weiteren Baukon- } \\
\text { struktionen }\end{array}$ & 4. Jh. v. Chr. - I. Jh. n. Chr. & $\begin{array}{l}\text { Chang/Grigoriev I999; } \\
\text { Baipakov 2008, 75-76 }\end{array}$ \\
\hline Cyganka 8567 & $\begin{array}{l}\text { fünf Grubenhäuser und mehrere dazuge- } \\
\text { hörige Speichergruben }\end{array}$ & 8.-I. Jh. v. Chr. & $\begin{array}{l}\text { Chang u.a. } 2003,30 \mathrm{I}-304 ; \\
\text { Baipakov } 2008,78\end{array}$ \\
\hline Turgen $2^{568}$ & $\begin{array}{l}\text { sechs abgerundete bzw. oval-rechteckige, } \\
\text { ebenerdige Häuser }\end{array}$ & 4.-3. Jh. v. Chr. & $\begin{array}{l}\text { Mar'jašev/Gorjačev 2009a, } \\
3-4,6-7,49-60,64-67 \\
\text { Mar'jašev/Gorjačev 2009b, } \\
\text { 3-4, I0-36 }\end{array}$ \\
\hline
\end{tabular}

putzten Flechtwerkwänden erbaut worden waren. Die Form des Daches blieb unklar (Baipakov 2008, $80-83)$.

Innerhalb von Hausbefunden der ersten Verbreitungszone wurden Feuerstellen festgestellt, die in einigen Fällen durch Steinsetzungen markiert waren. Auf den Böden von Haus- und Speichergruben kamen Phytolithen von Gerste, Überreste von Hirsehülsen und verschiedene Tierknochen zum Vorschein (Baipakov 2008, 75-79).

Die paläozoologischen Untersuchungen aller drei Niederlassungen der ersten Verbreitungszone ergaben, dass knapp die Hälfte des Tierbestandes Schaf/Ziege ausmachten. Dann folgten Rind, Pferd und Hund. In einigen Fällen kamen noch einzelne Knochen domestizierter Esel und Kamele vor. Wild wurde durch Knochen von Rothirsch, Reh, Wildschwein, Fuchs oder Hasen repräsentiert. Der Wildanteil ist vergleichsweise gering (Chang u.a. 2003, 305; Malachov 2005; Parzinger 2006, 793; Baipakov 2008, 77, 79).

Ferner ergaben die paläobotanischen Untersuchungen von Proben aus der Siedlung Tuzusaj folgende Ergebnisse zur Getreide- und Pflanzennutzung: Die Einwohner dieser Siedlung konsumierten Weizen, Gerste, Weintrauben, Nüsse (Chang/Grigoriev I999, 405) und „möglicherweise sogar Reis“ (Parzinger 2006, 793; Baipakov 2008, 76-77).

Zum Fundrepertoire der früheisenzeitlichen Siedlungen der ersten Verbreitungszone gehörten Steingeräte, wie Reib- und Schleifsteine, Stößel und eierförmige Steine, sog. Bolas, Gegenstände aus Knochen, wie Astragale und Amulette, die entweder aus der Spitze des Maralgeweihes oder aus den Reißzähnen von Raubtieren gefertigt waren, und Keramik. Die Keramik ist durch Fragmente von Schüsseln, Tassen, Vorratsgefäßen, Krügen, Töpfen, kesselartigen Töpfen oder Kesseln aus Ton repräsentiert. Es fand sich sowohl handgemachte als auch scheibengedrehte Keramik. Sie war meist unver-

${ }_{5} 64$ Dazu siehe Kap. 4.I Zu den Fragen der absoluten Chronologie der frühen Eisenzeit, siehe Tabelle 9 und Appendix I. Die ${ }^{14} \mathrm{C}-$ Datierungen. Graphische Darstellungen der Datierungsergebnisse und Kalibration.

${ }_{5} 65$ Erste Verbreitungszone, siehe Verzeichnis der früheisenzeitlichen Fundstellen mit Quellennachweis. Die Untersuchungen sind noch nicht abgeschlossen.
566 Erste Verbreitungszone, siehe Katalog.

${ }_{567}$ Siehe Anm. ${ }_{5} 66$.

568 Zweite Verbreitungszone, siehe Verzeichnis der früheisenzeitlichen Fundstellen mit Quellennachweis. Die Untersuchungen sind noch nicht abgeschlossen. 
ziert, jedoch traten einige Rand- und Wandscherben mit wellenförmigen Ritzlinien, Kanneluren, Wülsten oder Nadelabdrücken auf (Mar'jašev/Gorjačev I999a, 23; Gorjačev 2004, 7-8; Gorjačev 2005, I2, Baipakov 2008, 75-79, 82-83).

Im Bereich der früheisenzeitlichen Siedlung des archäologischen Komplexes Butakty I kam Eisenschlacke zum Vorschein, was als ein Hinweis für Metallbearbeitung in der Siedlung verstanden werden kann.

Die Erforschung der Siedlungsflächen zeigte, dass diese Niederlassungen permanent über das ganze Jahr benutzt wurden. Zudem übten die Bewohner der Tuzusaj-Siedlung einerseits sowohl den Trockenfeldbau als auch die Bewässerungswirtschaft aus und andererseits die Viehweidenwirtschaft. Die Viehweidenwirtschaft und die sesshaften Tätigkeiten werden auch für die Bewohner der Siedlung Butakty I vermutet (Baipakov 2008, 76, 79, 88).

Die einzige früheisenzeitliche Siedlung, die in der zweiten Verbreitungszone zum Teil untersucht wurde, zeigte, dass hier nur ebenerdige Häuser vorkamen. Diese Konstruktionen hatten Ähnlichkeit mit den Bauten der Siedlung Butakty I, mit dem Unterschied, dass sie direkt auf der Oberfläche errichtet wurden und der Boden aus gestampftem Lehm bestand. Dieser Stampflehmboden war zusätzlich mit einem Lehm-Mörtel beschmiert, der mit rötlichen und gelblichen, mineralischen Zusätzen gefärbt worden war. Ein wesentlicher Unterschied zu allen Hauskonstruktionen der ersten Verbreitungszone bestand darin, dass in keinem ebenerdigen Haus eine Feuerstelle festgestellt wurde. Solche Feuerstellen lagen im Bereich der Siedlungsfläche des archäologischen Komplexes Turgen 2 nur außerhalb der Häuser. Wie die Ausgräber vermuteten, lagen sie im Bereich des angrenzenden Hofes (Mar'jašev/Gorjačev 2009a, 55-56; Mar'jašev/Gorjačev 2009b, 22-24). An einige Häuser wurden rechteckige bzw. trapezförmige Flures69 angebaut (Mar'jašev/Gorjačev 2009a, 56; Mar'jašev/Gorjačev 2009b, 24).

Im Bereich der Häuser und in unterschiedlichen Wirtschaftsgruben wurden Arbeitsgeräte, Knochen und Keramik geborgen. Die Arbeitsgeräte aus Stein und die keramischen Funde wiesen weitgehend Übereinstimmungen mit den Gegenständen der ersten Verbreitungszone auf. Zusätzlich kamen Näpfe zum Vorschein. Die Gefäße aus Turgen 2 wiesen sowohl flache als auch runde Böden auf (Mar'jašev/Gorjačev I999b, 37; Mar'jašev/Gorjačev 2009b, 24, 26, 28). Jedoch kam aus der Siedlung Turgen 2 eine neue Kategorie von Arbeitsgerät aus Knochen hinzu. Es handelte sich dabei um unterschiedliche Arbeitsgeräte für die Lederbearbeitung, wie verschiedene Glätter, Pfrieme, Messer aus Knochen oder Knochengriffe (Mar'jašev/Gorjačev 2009a, 57; Mar'jašev/Gorjačev 2009b, 22-28). Des Weiteren können auch Spinnwirtel aus Ton erwähnt werden (Mar'jašev/Gorjačev 2009b, 28).

Die untersuchten Tierknochen, die im Siedlungsbereich geborgen wurden, zeigten folgende Anteile: 60\% kleine Wiederkäuer, 25\% große Wiederkäuer, 8\% Pferd und 7\% Wild. Wild wurde durch Knochen von Hirschen, Rehen, Hasen und Raubtieren (Wölfe oder Hunde und Schneeleoparden) repräsentiert (Nurumov I999; Gorjačev 2004, 34; Mar'jašev/Gorjačev 2009a, 57; Tjut'kova 2009).

Die Charakteristik der ebenerdigen Häuser sowie die Platzierung der Feuerstellen außerhalb der Gebäude lassen vermuten, dass es sich bei der Siedlung des archäologischen Komplexes Turgen 2 um eine in den Sommermonaten von früheisenzeitlichen Nomaden genutzte Station handelte. Die Bewohner dieser saisonalen Siedlung nutzten in regelmäßigen Zeitabständen die hochgelegenen Weiden des Transili-Alatau für ihre Herden, bearbeiteten gleichzeitig Felle und Leder und jagten. Die im Siedlungsbereich geborgenen Spinnwirtel zeugten zudem von Wollverarbeitung.

569 Häuser I, 3 und 5. 
Insgesamt konnte festgestellt werden, dass in einer Linie entlang der nördlichen Berghänge des Transili-Alatau Siedlungen errichtet wurden. Östlich des Dorfes Teskensu wurden allerdings keine weiteren Siedlungsspuren festgestellt57o. Es wurden befestigte als auch unbefestigte Siedlungen dokumentiert. Die befestigten Wohnplätze wiesen im Zentrum entweder einen trapezförmigen Hügel mit Schutzmauern und Schutzwällen auf, die durch eine künstliche Nachbearbeitung der Erosionsschluchten entstanden sind, oder es handelte sich um viereckig bzw. quadratisch gebaute Schutzanlagen, sog. Turtkuls, die mit der letzten mittelalterlichen Nutzungsphase solcher Niederlassungen verbunden wurden. Fast alle Siedlungsplätze dieser Linie wiesen an der Oberfläche fast keine Spuren von Bauten auf. Einige untersuchte Ansiedlungen zeigten, dass es sich bei den unbefestigten Siedlungen um ganzjährig bewohnte Niederlassungen handelte. Die Bewohner bauten Halbgruben- und Grubenhäuser oder jurtenartige Behausungen und übten sowohl Ackerbau als auch Viehweidenwirtschaft aus.

Als weitere Siedlungsgebiete kommen die hochgelegenen Bergtäler des südöstlichen Raumes in Frage. In dem Hochgebirgstal Turgen befinden sich weitere Siedlungen, die offensichtlich im Zusammenhang mit dem alpinen Weideland oberhalb der Bergwaldgrenze stehen. Man stellte hier ausschließlich unbefestigte Ansiedlungen fest. Sie befanden sich in der Nähe von Bächen oder Wasserquellen, in kleinen Schluchten, die von zwei oder drei Seiten durch Berge geschützt sind. Oberflächliche Strukturen stellten sich als rechteckige Gruben oder im Berghang eingegrabene, planierte, rechteckige Flächen dar, die in einigen Fällen von einzelnen Steinen umgrenzt wurden. Die einzige zum Teil ausgegrabene Siedlung der Früheisenzeit zeigte, dass hier ebenerdige Hauskonstruktionen vorherrschten, die nur saisonal in den Sommermonaten benutzt wurden. Die Bewohner der Siedlung des archäologischen Komplexes Turgen 2 nutzten die hochgelegenen Sommerweiden für ihre Herden, bearbeiteten Felle, Leder und Wolle und gingen auf die Jagd.

Am Ostrand des Plateaus Kegen befindet sich eine dritte Siedlungskonzentration. Es handelte sich ausschließlich um unbefestigte Siedlungen, die ähnliche Baukonstruktionen an der Oberfläche aufwiesen wie in der zweiten Verbreitungszone. Zusätzlich kommen zu einem Viereck angeordnete Steinreihen der Subkonstruktionen von Rechteckhäusern oder ein Rechteck bildende Doppelsteinreihen vor, die eventuell als Fundamentreste der Doppelwandhäuser interpretiert werden können. Die Niederlassungen der dritten Verbreitungszone orientieren sich an ähnlichen Landschaftsmarkern wie die Siedlungsplätze der zweiten Verbreitungszone. Ein Unterschied liegt jedoch darin, dass sich keine Wasserquellen in unmittelbarer Nähe befinden. Die Frage nach der Wasserversorgung bleibt hier momentan ungeklärt.

Einige Gräberfelder haben einen Bezug zu nahegelegenen Siedlungsplätzen, die vermutlich zeitgleich existierten. Diese Annahme kann jedoch nur hypothetisch bleiben, da diese Fundorte nur unvollständig erforscht wurden und eine komplexe Ausgrabung von Nekropole und in der Nähe gelegener Siedlung als unbedingtes Forschungsdesiderat genannt werden muss. Eine enge Kommunikation zwischen den Siedlungen an der nördlichen Seite des Transili-Alatau ist gut vorstellbar, da die Siedlungen in einem Abstand von I km bis zu I9 km voneinander entfernt lagen. Es ist denkbar, dass diese Strecken

570 Weiter östlich wurden noch zwei Turtkule - Lavar (ALT 57Im N43 34.042 E78 05.432; Literatur: Nurmuchanbetov/Savel'eva I988, I5; Savel'eva I994, 48-49, 93-II5, I28, Abb. I3-I4; Bajpakov u.a. 2002, 30, Abb. 9-IO) und Cilik (ALT 596m N43 36.093 E78 15.025; Literatur: Gorodeckij I924, 7I-72/122/, Nr. 26; Bernštam I94Ib, 8; Pacevič I956, 26-3I; Ageeva I957, 54/54-56/58, 57/66;
Ageeva I96ob, 2-5, Abb. I-3; Nurmuchanbetov/ Savel'eva I988, I5-I6; Savel'eva I994, 50-52, 93-II5, I26, Abb. I6-I8, 35,3 und 40,2; Bajpakov u.a. 2002, 30-3I, Abb. I3, I08) festgestellt. Hier sprechen die aufgelesenen Keramikscherben für eine Datierung ins Mittelalter. 
zwischen zwei Niederlassungen zu Fuß, zu Pferde oder mit einem Karren an einem Tag zurückgelegt werden konnten. Durch die Platzwahl der Siedlungen entstand eine Art Linie, die bei der Stadt Almaty im Westen begann, entlang der nördlichen Bergrücken des Transili-Alatau $80 \mathrm{~km}$ nach Osten verlief und sich von dort nach Südosten bis ins Gebirge in Richtung Kirgisien (Issyk-Kul See) und möglicherweise noch weiter südwestlich oder südlich in Richtung Iran und Indien fortsetzte. Hervorzuheben ist, dass sich Siedlungen, die als befestigt erscheinen, nur an der nördlichen Seite des Transili-Alatau befinden. In den hochgelegenen Gebirgstälern sind solche Ansiedlungen stets unbefestigt. Dies könnte möglicherweise mit der ständig drohenden Gefahr im Flachland bzw. der offenen Steppe erklärt werden.

Die befestigten Siedlungen, die flächenmäßig etwas größer als die unbefestigten der ersten Verbreitungszone waren, könnten, wie es von K. M. Bajpakov vermutet wurde (Baipakov 2008, 72), theoretisch als mögliche Herrschaftssitze und die nahegelegenen Großkurgane als Begräbnisstätten der sakischen Fürsten gedient haben. Allerdings bleibt offen, inwieweit die befestigten Siedlungen jüngerer Perioden mit sakenzeitlichen Siedlungsspuren tatsächlich als mögliche Herrschaftssitze sakischer Fürsten interpretiert werden können, da noch nicht eindeutig belegt ist, ob diese Fundorte während der Früheisenzeit bereits befestigt waren. In den meisten Fällen ist vorstellbar, dass die Niederlassungen Schutzorte für die sesshafte Bevölkerung des südöstlichen Siebenstromlandes waren und als Anlaufstellen für Karawanen dienten. Nicht alle Vertreter der Elite, die vermeintlich in den Großkurganen bestattet wurden, hatten offensichtlich einen Wohnsitz im Untersuchungsgebiet, da der großen Zahl der Gräberfelder mit großen Kurganen keine vergleichbare Anzahl befestigter Siedlungen entspricht (Siedlungen Rachat und Krasnyj Vostok). Möglicherweise befanden sich die Residenzen der Fürsten in vom Bestattungsplatz weit entfernten Gebieten, was jedoch einen überregionalen Machtanspruch nicht ausschließen muss. Wer und wann tatsächlich diese Siedlungsplätze bewohnte, muss offen bleiben, da der heutige Wissensstand im Bezug auf das Siedlungswesen im Untersuchungsgebiet große Lücken aufweist. De facto bleibt festzuhalten, dass im südöstlichen Siebenstromland Siedlungen unterschiedlicher Größe existierten. Weiterführende Fragen nach den tatsächlichen Bewohnern, der genauen Zeitstellung der Wohnplätze und nach Entwicklung und Dynamik des Siedlungswesens bleiben zunächst offen. In den unweit einiger Siedlungsplätze gelegenen Großkurganen wurde die sakische Elite bestattet, die zu den Reiterkriegernomaden gehörte. Zur Kultur der sesshaften Bevölkerung konnten anhand der Untersuchungen der Nekropolen mit solchen Kurganen keine Aussagen getroffen werden. Für die Siedlungsarchäologie der Früheisenzeit konnte ein enormer Forschungsbedarf für das Arbeitsgebiet aufgezeigt werden. 